\title{
Long noncoding RNAs in human disease: emerging mechanisms and therapeutic strategies
}

\begin{abstract}
"Although the antisense oligonucleotide technology seems most promising for therapy, other approaches should be explored as well..."
\end{abstract}

Keywords: disease $\bullet$ epigenetic $\bullet$ genomic imprinting $\bullet$ long noncoding RNA

With the advent of next-generation sequencing technologies, there is a rapidly growing interest in long noncoding RNAs (lncRNAs). This class of processed RNA is defined as molecules with a length greater than 200 nucleotides and with no apparent coding potential. More than 10,000 lncRNAs were discovered in humans and in mice during the last years [1]. To account for their considerable heterogeneity, different subclassifications have been proposed, depending for instance on the molecular properties or the subcellular localization of the lncRNA. Although, so far, only few IncRNAs have been functionally characterized, their evolutionary conservation and specific expression patterns suggest biological relevance for many.

Long ncRNAs are particularly important in the control of gene expression, at the transcriptional, the posttranscriptional and also the translational level. Strong evidence for this has come from lncRNAs involved in the developmental expression of homeotic genes [2], from the lncRNA Xist (' $X$ inactive-specific transcript'), which controls $\mathrm{X}$ chromosome inactivation in females [3], and from lncRNAs involved in the mono-allelic expression of imprinted genes [4]. In these well-studied examples, the IncRNA is retained in the nucleus and mediates repressive or activating chromatin alterations, and thereby stably controls gene expression in cis [5]. Recent research on the imprinted Prader-Willi syndrome (PWS) disease locus on human chromosome 15q11q13 reveals that lncRNAs can exert effects in trans as well, on gene loci located elsewhere in the genome [5,6]. Moreover, a muscle-specific study pinpointed different lncRNAs that encode micropeptides [7]. This novel concept not only enlarges the functional spectrum but also challenges the basic definition of lncRNA.

Several lncRNAs are now known to be causally involved in human disease $[1,8]$. Of particular interest are the DBE-T IncRNA involved in facioscapulohumeral muscular dystrophy (FSHD), an RNA which regulates histone modifications in cis, and the lncRNA UBE3A-ATS perturbed in the imprintingrelated Angelman Syndrome (AS), which acts through transcriptional interference. Several recent studies have explored how these lncRNAs may act and evoke possible therapeutic avenues to alleviate these diseases.

FSHD is an autosomal dominant disease that maps to chromosome 4q35. In the patients, several protein-coding genes at the locus are transcriptionally activated in an aberrant manner. Genetically, FSHD is caused by a partial loss of a tandemly repeated DNA element, the so-called D4Z4 repeat. Till recently, the link between the decreased D4Z4 repeat size and the deregulation of close-by genes was unknown. The group of Davide Gabellini discovered that an lncRNA is involved in this process [9]. In healthy individuals, the large D4Z4 repeat is bound by Polycomb repressive complexes (PRCs), which bring about histone H3-lysine-27 trimethylation (H3K27me3). This repressive chromatin mark, together with an inactive conformation of the domain, ensures the repression of neighboring genes at the locus. Upon reduction of the number of D4Z4 repeats in FSHD patients, however,
Sébastien Lalevée

Institute of Molecular Genetics (IGMM), Centre National de Recherche Scientifique (CNRS), 1919 Route de Mende, 34293 Montpellier, France and

The University of Montpellier, 163 rue Auguste Broussonnet, 34090 Montpellier, France

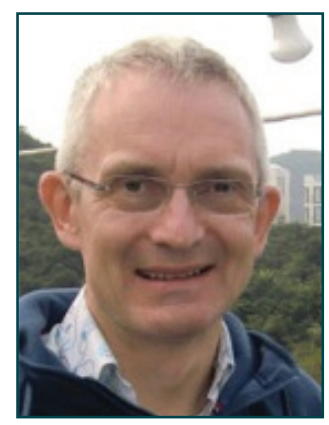

Robert Feil

Author for correspondence: Institute of Molecular Genetics (IGMM), Centre National de Recherche Scientifique (CNRS), 1919 Route de Mende, 34293 Montpellier, France and

The University of Montpellier, 163 rue Auguste Broussonnet, 34090 Montpellier, France Tel.: +33 434359663 robert.feil@igmm.cnrs.fr 
an IncRNA named DBE-T is produced from the few remaining, no longer silenced, repeats. DBE-T RNA interacts with ASH1L, a component of the Trithorax chromatin activator complex, which leads to the deposition of activating H3 lysine-36 dimethylation (H3K36me2) at the locus. This 'opening up' of the chromatin derepresses the close-by protein-coding genes which likely contribute to the etiology of the disease [9].

FSHD provided the first evidence of IncRNA-mediated gene activation in human disease. Although DBET's precise involvement in the derepression of genes is still unclear, it is likely that the lncRNA itself plays a key role in the process. DBE-T should, therefore, be an attractive target for future therapeutic research.

AS is a complex neurological disorder that maps to the same imprinted chromosome 15q11-q13 region as the neurological syndrome PWS [8]. It is caused by a loss of expression of the $U B E 3 A$ gene, most often as a consequence of genetic mutations. UBE3A encodes an essential E3 ubiquitin ligase and is imprinted in neuronal cells, with expression occurring from the maternally inherited chromosome exclusively. On the paternal chromosome, $U B E 3 A$ is completely repressed. What controls this paternal UBE3A repression? At the locus, an IncRNA called UBE3A-ATS ('UBE3AAntisense Transcript') is specifically expressed from the paternal chromosome and overlaps in its 3'-portion with the $U B E 3 A$ gene. Interestingly, the Ube3aats IncRNA remains localized at its site of transcription [10]. Elegant mouse studies showed that truncation of the lncRNA, through an inserted poly-A terminator, was sufficient to induce expression of UBE3A on the paternal chromosome [10]. It has been proposed, therefore, that the IncRNA Ube3a-ats controls Ube3a mRNA expression via a transcriptional interference mechanism, leading to the repression of the paternal Ube3a allele in cis on the paternal chromosome. As a consequence of different genetic mutations in AS, either the IncRNA UBE3A-ATS becomes expressed biallelically and this abrogates $U B E 3 A$ expression on both parental chromosomes, or the $U B E 3 A$ gene itself becomes nonfunctional leading to a loss of protein expression.

The involvement of lncRNAs in AS, FSHD and other human diseases provides interesting opportunities for new therapeutic strategies, notably via the targeting of the lncRNA itself. Exciting recent studies on AS indicate how this might be achieved.

Given the apparently direct mode of action of UBE3A-ATS, considerable efforts have been directed toward reactivation of $U B E 3 A$. As a tool for the screening of drugs, a knock-in mouse line was created in which the paternal $U B E 3 A$ gene was tagged with a fluoro- phore (YFP) expressing sequence. In primary neuronal cells, fluorescence was then used as a sensitive read-out for re-expression of the paternal $U B E 3 A$ gene. In a first strategy, a library of small compounds was screened for possible re-expression of UBE3A [11]. Among the best hits was a topoisomerase inhibitor called irinotecan. Further research demonstrated that all topoisomerase inhibitors tested reactivated the paternal Ube $a$ allele. Topotecan, another specific topoisomerase inhibitor, was the most potent compound among all drugs tested. In parallel to the reactivation of the paternal $U B E 3 A$ gene, a decrease in Ube3a-ats expression occurred in the Topotecan-treated cells. Topotecan's mechanism of action has since been explored further. Inhibition of Ube3a-ats transcriptional elongation and R-loop (DNA-RNA) structures were thus found to play an important role in the allelic repression of Ube3a [12,13]. In subsequent mouse studies, significantly, intracerebroventricular and intrathecal injections of Topotecan were shown to have sustained effects over a period of several months [11].

Using the same fluorescent YFP reporter system, an even more straightforward approach was employed, which could be less toxic in vivo than Topotecan. The Ube3a-ats lncRNA itself was directly targeted using an array of second-generation antisense oligonucleotide (ASOs) [14]. These phosphorothioate-modified chimaeric 2'-O-methoxyethyl DNA oligonucleotides have the propensity to target nuclear lncRNAs with great efficiency [15]. In neuronal cells, the carefully designed ASOs targeted Ube3a-ats in the nucleus and this induced a re-expression of the Ube3a transcripts, similarly as observed in the Topotecan treatments. In an AS mouse model, a single intracerebroventricular injection of ASOs was sufficient to decrease Ube3a-ats expression in the brain and markedly activated $U B E 3 A$ expression at both the RNA and protein level [14]. Concordantly, ASO treatment improved the body weight and the behavior of the AS mice. These encouraging outcomes were sustained for more than 4 months postinjection, demonstrating the long-term benefits of this novel oligonucleotide-based therapeutic approach.

Admittedly, we do not yet understand well the mechanism(s) of action of ASOs, their specificity or their possible long-term effects. Nevertheless, this novel oligonucleotide approach might be applied as an alternative to, or in combination with, already US FDA-approved drugs. IncRNA-targeting ASOs have not been applied in humans so far. However, two commercial mRNA-targeting ASOs, Fomirvisen and Mipomersen, already have FDA approval for the treatment of cytomegalovirus infection and hypercholesterolaemia, respectively [16,17]. Different ASO-based Phase II and Phase III clinical trials have also been 
launched for the treatment of cancer, graft rejection, as well as for rare diseases including Crohn's disease. These multicenter clinical studies corroborate the ASO-associated low cytotoxicity, and the long-term beneficial effects observed in mice suggest that this technology might be rapidly transferred to the clinic for IncRNA-associated diseases. In imprinting disorders other than AS, including PWS, Beckwith-Wiedemann syndrome and Kagami-Ogata syndrome, nuclear lncRNAs are mechanistically linked to the disease as well $[5,8]$.

A challenge for the coming years will be to further improve the design of ASOs and develop strategies for their efficient delivery and site-specific targeting. Future improvements of the ASO technology will surely be beneficial to fundamental research as well, for instance, into physiological and developmental roles of the many $\operatorname{lncRNAs}$ whose functions are still enigmatic.

\section{References}

1 Sun M, Kraus WL. From discovery to function: the expanding roles of long noncoding RNAs in physiology and disease. Endocr. Rev. 36(1), 25-64 (2015).

2 Rinn JL, Kertesz M, Wang JK et al. Functional demarcation of active and silent chromatin domains in human HOX loci by noncoding RNAs. Cell 129(7), 1311-1323 (2007).

3 Brown CJ, Ballabio A, Rupert JL et al. A gene from the region of the human $\mathrm{X}$ inactivation centre is expressed exclusively from the inactive $\mathrm{X}$ chromosome. Nature 349(6304), 38-44 (1991).

4 Barlow DP, Bartolomei MS. Genomic imprinting in mammals. Cold Spring Harb. Perspect. Biol. 6(2), pii: a018382 (2014).

5 Sanli I, Feil R. Chromatin mechanisms in the developmental control of imprinted gene expression. Int. J. Biochem. Cell Biol. doi:10.1016/j.biocel.2015.04.004 (2015) (Epub ahead of print).

6 Stelzer Y, Sagi I, Yanuka O, Eiges R, Benvenisty N. The noncoding RNA IPW regulates the imprinted DLK1-DIO3 locus in an induced pluripotent stem cell model of PraderWilli syndrome. Nat. Genet. 46(6), 551-557 (2014).

7 Anderson DM, Anderson KM, Chang CL et al. A micropeptide encoded by a putative long noncoding RNA regulates muscle performance. Cell 160(4), 595-606 (2015).

8 Girardot M, Feil R, Lleres D. Epigenetic deregulation of genomic imprinting in humans: causal mechanisms and clinical implications. Epigenomics 5(6), 715-728 (2013).

9 Cabianca DS, Casa V, Bodega B et al. A long ncRNA links copy number variation to a polycomb/trithorax epigenetic switch in FSHD muscular dystrophy. Cell 149(4), 819-831 (2012).

10 Meng L, Person RE, Huang W, Zhu PJ, Costa-Mattioli M, Beaudet AL. Truncation of Ube3a-ATS unsilences paternal Ube3a and ameliorates behavioral defects in the Angelman
Mass spectrometry-based technologies can be applied to identify the regulatory proteins that bind to specific lncRNAs, as was elegantly shown in recent studies on the Xist lncRNA involved in X-inactivation [18,19]. Although the ASO technology seems most promising for therapy, other approaches should be explored as well, particularly in case a pathological loss of lncRNA expression needs to be corrected [20].

\section{Financial \& competing interests disclosure}

The authors have no relevant affiliations or financial involvement with any organization or entity with a financial interest in or financial conflict with the subject matter or materials discussed in the manuscript. This includes employment, consultancies, honoraria, stock ownership or options, expert testimony, grants or patents received or pending, or royalties.

No writing assistance was utilized in the production of this manuscript

Syndrome mouse model. PLoS Genet. 9(12), e1004039 (2013).

11 Huang HS, Allen JA, Mabb AM et al. Topoisomerase inhibitors unsilence the dormant allele of $U B E 3 A$ in neurons. Nature 481(7380), 185-189 (2012).

12 Powell WT, Coulson RL, Gonzales ML et al. R-loop formation at Snord116 mediates topotecan inhibition of Ube3a-antisense and allele-specific chromatin decondensation. Proc. Natl Acad. Sci. USA 110(34), 13938-13943 (2013).

13 King IF, Yandava CN, Mabb AM et al. Topoisomerases facilitate transcription of long genes linked to autism. Nature 501(7465), 58-62 (2013).

14 Meng L, Ward AJ, Chun S, Bennett CF, Beaudet AL, Rigo F. Towards a therapy for Angelman Syndrome by targeting a long non-coding RNA. Nature 518(7539), 409-412 (2015).

15 Wheeler TM, Leger AJ, Pandey SK et al. Targeting nuclear RNA for in vivo correction of myotonic dystrophy. Nature 488(7409), 111-115 (2012).

16 Orr RM. Technology evaluation: Fomivirsen, Isis Pharmaceuticals Inc/CIBA vision. Curr. Opin. Mol. Ther. 3(3), 288-294 (2001).

17 Raal FJ, Santos RD, Blom DJ et al. Mipomersen, an apolipoprotein B synthesis inhibitor, for lowering of LDL cholesterol concentrations in patients with homozygous familial hypercholesterolaemia: a randomised, double-blind, placebo-controlled trial. Lancet 375(9719), 998-1006 (2010).

18 Mchugh CA, Chen CK, Chow A et al. The Xist lncRNA interacts directly with SHARP to silence transcription through HDAC3. Nature 521(7551), 232-236 (2015).

19 Chu C, Zhang QC, Da Rocha ST et al. Systematic discovery of Xist RNA binding proteins. Cell 161(2), 404-416 (2015).

20 Bassett AR, Akhtar A, Barlow DP et al. Considerations when investigating lncRNA function in vivo. Elife 3, e03058 (2014). 